\title{
Optimus: SINR-driven Spectrum Distribution via Constraint Transformation
}

\author{
Lili Cao, Lei Yang, Xia Zhou, Zengbin Zhang, Haitao Zheng \\ Department of Computer Science, University of California, Santa Barbara
}

\begin{abstract}
How to distribute radio spectrum across network nodes is a critical problem in spectrum auctions and management. In this paper, we consider the problem of distributing spectrum using SINR-driven physical interference models. We propose Optimus, a new line of approximation algorithms that perform within a constant distance of $\min \left\{2^{\alpha}+1,10\right\}$ from the optimum in terms of spectrum usage efficiency, where $\alpha \geq 2$ is the pathloss exponent. Different from conventional greedy solutions, Optimus applies a global optimization mechanism that transforms the spatial interference constraints into a set of linear constraints, reducing the original optimization into a linear/convex/separable programming problem. While linearization techniques have been applied in prior works, Optimus makes a new and important contribution by deriving a highly efficient constraint transformation applicable to general network configurations. Experiments using real network measurements and sophisticated propagation models show that Optimus outperforms existing solutions by $20-50 \%$ in spectrum utilization and is within $20 \%$ gap from the optimum. Optimus supports a wide variety of objective functions, and is applicable to many spectrum-driven applications such as spectrum auctions and spectrum admission control.
\end{abstract}

\section{INTRODUCTION}

The fast, organic growth of wireless networks has created a challenging problem: "how to distribute radio spectrum among network nodes on-demand while avoiding harmful interference?" This spectrum distribution problem differs from conventional channel allocation problems because the amount of spectrum assigned to each network node is often unknown and must be determined during the optimization [1]. Solving this problem is an integral part of emerging spectrum related applications including dynamic spectrum auctions [1$4]$ and management [5-7].

The majority of existing proposals take an indirect approach: first simplify physical interference conditions into a set of pairwise constraints (or the graph interference model), then distribute spectrum on top of them (or the graph model) [1,3-8]. Such simplification, however, comes at an unpleasant cost. Radio interference is inherent accumulative and cannot be accurately represented by pair-wise constraints. As a result, decisions made on top of the graph model could lead to inefficient allocation or unwanted interference. Similar pitfall was also identified in other networking problems such as link scheduling [9-11].

In this paper, we consider the problem of distributing spectrum across many network nodes under physical interference models. Consider a dynamic spectrum auction system [1] where access points (from the same and different networks) request spectrum from a central authority. The authority determines the winners and their spectrum usage to maximize a predefined system utility, e.g. social welfare or auction revenue. Different from cellular networks $[12,13]$, the node deployment is unplanned and nodes' coverage areas often overlap. Their interference conditions depend heavily on local node density and vary significantly across geographic locations.

Such unregulated topology introduces new challenges to spectrum distribution, posing a need for new solutions. First, most existing solutions take a greedy approach $[14,15]$ : allocate spectrum to nodes sequentially, making the locally optimal choice at each step. It works well under graph models or regulated topology because the impact of a local decision can be clearly identified. Under unregulated topology and physical models, the impact of interference is accumulative and hard to predict. Thus the algorithm can easily fall into local optimum too early, making it hard to provide good approximation guarantees. Second, other works have developed efficient solutions by transforming the problem into linear/convex programming. These solutions, however, must assume a regulated topology $[12,13]$, or simplified interference models $[1,16-19]$. Under unregulated topology and physical models, existing transformations no longer apply and there is no trivial extension. Finally, the impact of unregulated topology has been considered in link scheduling and channel allocation $[10,20,21]$. The proposed solutions, however, assume the amount of resource (slots or channels) assigned to each link is known and fixed, and thus cannot be applied to the problem of spectrum distribution we target in this paper. 
To address these challenges, we propose Optimus, a new framework for distributing spectrum on top of the SINR-based physical interference model. By transforming the interference constraints into linear constaints on nodes' spectrum usage, Optimus successfully reduces the original NP-hard optimization problem into a variant of linear/convex programming problem. Our key contribution is to discover an efficient linear transformation of the physical interference condition of any arbitrary node topology. We also prove analytically that this transformation leads to a feasible allocation whose spectrum usage efficiency is within a constant bound from the optimum.

Aside from theoretical analysis, we also evaluate Optimus using extensive network simulations. Because its performance depends heavily on the underlying radio propagation condition, we consider three different propagation environments: a standard geometric propagation model, a sophisticated RF mapping software which accounts for the terrain effect, and finally a collection of measured RF signal traces from the Mountain View Google WiFi network. These results consistently demonstrate Optimus's efficiency and its improvement over existing solutions.

Overall, Optimus provides the following benefits:

- Constant performance bound - Under geometric signal propagation, Optimus performs within a constant $\left(\min \left\{2^{\alpha}+1,10\right\}\right)$ bound from the optimum in terms of spectrum usage efficiency, where $\alpha \geq 2$ is the pathloss exponent. Prior solutions [2, $11,20]$ become unbounded as network size increases or as $\alpha$ changes.

- High efficiency - Our network simulation results show that Optimus is within 10-20\% gap to the optimal solution derived via exhaustive search. Compared to conventional greedy algorithms, Optimus achieves an average gain of $30 \%$ across a large set of topologies. In certain cases, its gain can be up to $300 \%$.

- Applicable to many optimization objectives - Optimus and its analytical bounds apply to a variety of general utility functions. Depending on the utility functions, the central authority can apply different optimization techniques.

- Applicable to arbitrary network topology Optimus and its analytical bounds apply to any arbitrary network topology. It captures the impact of accumulative interference rather than that of the most dominant interferer [19].

Applications of Optimus. As a specialized solution for spectrum distribution, Optimus can be directly plugged into existing frameworks on dynamic spectrum auctions $[1,4]$ and spectrum management [7]. However,

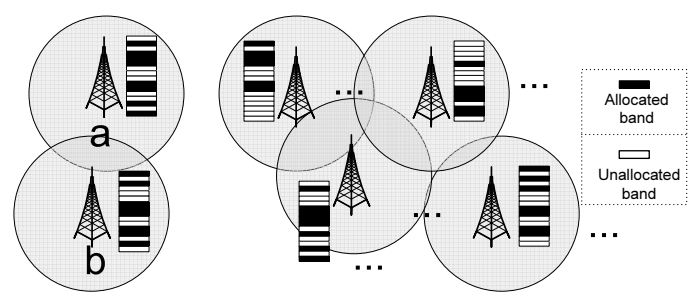

Figure 1: A set of network nodes request spectrum usage, each can use a flexible set of noncontiguously aligned frequency bands. The problem is to decide how many and which bands each node receives to maximize a system utility.

Optimus is designed under typical assumptions of spectrum distribution, and thus shall not be applied to other specialized problems. For example, Optimus requires the available spectrum to be finely partitioned into a large number of homogeneous bands, which is typical in spectrum distribution but not in WiFi channel allocation. Optimus assumes that the interference condition is modeled by the average or worst case SINR and thus does not consider channel fading, time variation or capacity constraints.

\section{PRELIMINARIES}

In this section, we define the problem of spectrum distribution under the SINR-driven physical interference model. We show that this problem differs significantly from link scheduling or conventional channel allocation problems.

As shown in Figure 1, we consider the spectrum distribution/auction problem defined by $[1,3]$. A set of $K$ network nodes request spectrum from a central authority to support their downlink transmissions to their users. The spectrum is finely partitioned into a large number $(M>>1)$ of frequency bands, and the bands are of the same width and experience the same statistical propagation conditions. Each network node can accept multiple bands even if they are non-contiguously aligned in frequency. The objective is to assign bands among network nodes to maximize a predefined system utility subject to the interference constraints. Because bands are homogeneous, the utility function depends on the amount of "successful" spectrum usage each node obtains.

Definition 1. Let $\mathbb{A}$ define a spectrum allocation,

$$
\mathbb{A}=\left\{a_{m, i}\right\}_{1 \leq m \leq M, 1 \leq i \leq K},
$$

where $a_{m, i}=1$ indicates that node $i$ receives band $m$, otherwise $a_{m, i}=0$.

Definition 2. Under the SINR-based physical interference model [22], a spectrum allocation $\mathbb{A}$ is successful if on each band $m$ allocated to a node $i$, at any location related to i's network operation, the received signal to 
interference and noise ratio is above a threshold $\beta$ :

If $a_{m, i}=1$, then $S I N R_{m, i} \geq \beta, \forall m \in[1, M], i \in[1, K]$

The value of $S I N R_{m, i}$ depends on the specific network scenario and the targeted interference model, but it generally follows the following format:

$$
S I N R_{m, i} \triangleq \frac{S_{i}}{\sum_{j \neq i} a_{m, j} \cdot I_{j, i}+N} .
$$

In our scenario, $S I N R_{m, i}$ is the minimum SINR on band $m$ across all the points in $i$ 's coverage area. In this case, $S_{i}$ is the minimum received signal strength across $i$ 's coverage area, $I_{j, i}$ is the maximum interference strength a node $j$ can produce to any receiver in $i$ 's coverage area, and $N$ is the noise level. More specifically, let $P_{i}$ denote $i$ 's transmit power, $Q_{j, i}(j \neq i)$ denote the smallest pathloss from node $j$ to any receiver in $i$ 's coverage area, and $Q_{i, i}$ denote the maximum pathloss from $i$ 's transmitter to any receiver in its own coverage area. Then we have $S_{i}=P_{i} / Q_{i, i}$ and $I_{j, i}=P_{j} / Q_{j, i}$.

Definition 3. Let $V_{i}$ represent the amount of spectrum a node $i$ obtains from a successful spectrum allocation: $V_{i}(\mathbb{A})=\sum_{m} a_{m, i}$ if $\mathbb{A}$ is successful. A volume vector $\mathbf{V}=\left\{V_{1}, V_{2}, \ldots, V_{K}\right\}$ is achievable if it can be achieved by some successful spectrum allocation.

Definition 4. A spectrum distribution problem is to find a successful allocation $\mathbb{A}$ and its $\mathbf{V}$ to maximize a system utility $\mathbb{U}(\mathbf{V})=\mathbb{U}\left(V_{1}, V_{2}, \cdots, V_{K}\right)$.

Example utilities include spectrum utilization $\mathbb{U}(\mathbf{V})=$ $\sum_{i=1}^{K} V_{i}$, auction revenue $\mathbb{U}(\mathbf{V})=\sum_{i=1}^{K} V_{i} \cdot b_{i}\left(V_{i}\right)$ where $b_{i}($.$) is the unit price, and proportional fairness \mathbb{U}(\mathbf{V})=$ $\sum_{i=1}^{K} \log V_{i}$.

This definition shows that the spectrum distribution problem differs significantly from link scheduling, because the amount of spectrum assigned to a node $V_{i}$ is unknown prior to the optimization and must be determined during the optimization. Therefore, although existing works on link scheduling have developed solutions within provable bounds $[10,20,21,23]$, they are not applicable to the spectrum distribution problem we target in this paper.

\section{A CASE FOR CONSTRAINT TRANSFOR- MATION}

In this section, we present the high-level design principles behind Optimus. We start by examining existing solutions for spectrum distribution under both physical and graph models. Interestingly, most existing solutions use greedy allocations, and have developed efficient algorithms that provide tight approximation bounds under graph interference models $[6,24]$. In general, a greedy strategy starts from an empty allocation and allocates bands to nodes sequentially. To each node, the algorithm decides how many and which bands to allocate, assuring that each new allocation will not make the SINRs of allocated nodes fall below $\beta$. The algorithm stops when no more allocation can be made.

Using two examples, we show that greedy solutions are not well-suited for distributing spectrum under physical interference models. The first example applies the above greedy solution to the network in Figure 1. It first assigns band 1 to both node $a$ and $b$, making their SINRs very close to $\beta$. Now it cannot assign the same band to any other distant nodes without disrupting $a$ or $b$ 's SINR. But if the algorithm had not assigned the band to $b$, it could assign the band to more than two additional nodes. This example shows that because interference is accumulative, it is extremely hard to predict the interference a node receives from future allocation stages. Without this information, the algorithm is likely to make suboptimal decisions early in the process, and cannot reach a good solution.

In the second example we consider distributing spectrum to maximize node fairness. Using greedy allocation, it is difficult to determine the number of bands assigned to each node - assigning too many or too few bands to nodes will both degrade the fairness. This example shows that the problem of distributing spectrum is different from the link scheduling problem where the amount of resource assigned to each node is predefined. For our spectrum distribution problem, the number of bands each node should receive is an optimization parameter depending on the global utility, and thus cannot be determined locally.

From the above two examples, we also observe two key design principles:

- Consider network-wide accumulative interference: When allocating spectrum to a node, the algorithm should consider the potential accumulative interference to and from the entire network.

- Consider network-wide node spectrum usage: When determining the amount of spectrum allocated to each node, the algorithm should jointly consider the network-wide $\left\{V_{i}\right\}_{1 \leq i \leq K}$ in order to maximize the system utility.

Constraint Transformation. The above two design principles push for a global planning technique. An ideal solution is constraint transformation. The original spectrum distribution is hard because of the complex interference constraints. But if we can transform them into a set of simpler constraints, we can find a feasible solution (at the cost of some performance degradation from simplifying the constraints).

We illustrate this concept using a simple example consisting of two network nodes $a$ and $b$. Suppose $a$ and $b$ 's mutual interference is strong enough that they must use different spectrum. In this case, the impact of interference can be represented by $V_{a}+V_{b} \leq M$ and any $V_{a}$ 
and $V_{b}$ satisfying this constraint can be realized using a successful allocation. Now if the goal is to allocate spectrum to $a$ and $b$ to maximize a revenue defined by: $R=2 V_{a}+V_{b}$, then the solution is $V_{a}=M, V_{b}=0$; and if the goal is to maximize fairness, then the solution is $V_{a}=V_{b}=M / 2$.

The concept of constraint transformation has been applied to resource allocation problems in cellular networks. Existing solutions, however, must assume a simplified physical interference model such as using a regulated topology $[12,13]$, or only considering a single dominant interferer for each node [19], or applying a graph interference model [16]. Therefore, a key challenge in this paper is to define a set of linearized interference constraints to best represent the complex physical interference model under arbitrary network topologies.

\section{OPTIMUS}

Using the concept of constraint transformation, we present Optimus, a new framework of spectrum distribution under the SINR-driven interference model. Optimus applies a global planning technique - it transforms the interference constraints into a set of linear constraints on the volume vector $\mathbf{V}=\left(V_{1}, V_{2}, \cdots, V_{K}\right)$, reducing the original $\mathrm{NP}$-hard problem into a tractable problem. Optimus's key contribution is to define a set of linearized interference constraints from the complex physical interference model under arbitrary network topology.

One requirement of Optimus is that the worst case pathloss $Q_{i, j}=Q_{j, i}$, so that the allocation algorithm will converge. For cases where $Q_{i, j} \neq Q_{j, i}$, Optimus replaces $Q_{i, j}$ and $Q_{j, i}$ with $\max \left\{Q_{i, j}, Q_{j, i}\right\}$ so that its solution will not violate the original SINR requirement. In Section 6 we use measured RF signal traces to evaluate the impact of this requirement.

\subsection{The Linearized Interference Constraints}

Finding a linearized representation of the physical interference condition is highly challenging, especially when the network topology is unregulated and contains a large number of nodes. To avoid harmful interference and unnecessary loss of efficiency, we must judiciously define the new constraints. They must be stricter than the original ones so that the corresponding $\mathbf{V}$ is achievable by a successful allocation. They must account for the accumulative interference across the network, and introduce as little performance degradation as possible. They should ideally be linear constraints so that efficient algorithms like linear/convex programming can be applied to find the utility-maximizing $\mathbf{V}$.

With these requirements in mind, Optimus replaces the original physical interference constraints defined in Section 2:

If $a_{m, i}=1$, then $S I N R_{m, i} \geq \beta, \forall m \in[1, M], i \in[1, K]$ with the following linearized constraints on $\mathbf{V}$ :

$$
\begin{array}{r}
V_{i}+\sum_{j \neq i} V_{j} \cdot \frac{I_{j, i}^{+}}{I_{i}^{\text {max }}} \leq M, \quad \forall_{1 \leq i \leq K} \\
\text { where } I_{i}^{\max }=\frac{S_{i}}{\beta}-N, \quad I_{j, i}^{+}=\min \left(I_{i}^{\max }, I_{j, i}\right)
\end{array}
$$

These constraints are driven by a local adjustment algorithm that assigns spectrum bands to nodes to minimize their perceived interference. In the following, we introduce a theorem that validates the new interference constraints, and the local adjustment algorithm. The same algorithm will be used in Optimum for allocating spectrum bands among nodes after finding the utilitymaximizing $\mathbf{V}$.

Theorem 1. If $\mathbf{V}=\left(V_{1}, V_{2}, \cdots, V_{K}\right)$ satisfies the constraints defined in (2) then $\mathbf{V}$ can be achieved by a successful spectrum allocation.

Proof. We prove Theorem 1 by describing the allocation algorithm that finds a successful allocation for each $\mathbf{V}$ that satisfies the constraints. As shown in Algorithm 1 , each node $i$ iteratively selects $V_{i}$ bands with the smallest aggregated interference, and repeats the process until no adjustments can reduce the interference at any node. We show that Algorithm 1 achieves the following two properties, which directly imply Theorem 1. The proofs of Lemma 1 and 2 are in the Appendix.

Lemma 1. Algorithm 1 will converge.

Lemma 2. Each $\mathbf{V}$ satisfying the condition defined by (2) can be achieved by a successful allocation using Algorithm 1.

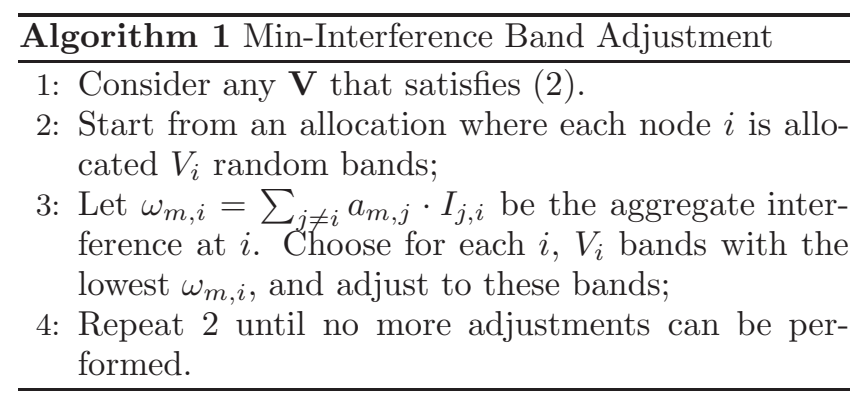

\subsection{The New Optimization Problem}

With the new linear constraints, the original utilitymaximization problem reduces to

$$
\max _{\mathbf{V}} \mathbb{U}(\mathbf{V})=\mathbb{U}\left(V_{1}, V_{2}, \cdots, V_{K}\right)
$$

subject to

$$
V_{i}+\sum_{j \neq i} V_{j} \cdot \frac{I_{j, i}^{+}}{I_{i}^{\text {max }}} \leq M, \quad \forall_{1 \leq i \leq K} .
$$




\begin{tabular}{|c|l|}
\hline Utility Function & Solution \\
\hline Spectrum utilization & $\mathbb{U}(\mathbf{V})=\sum_{i=1}^{K} V_{i}$, linear programming, \\
\hline Proportional fairness & $\mathbb{U}(\mathbf{V})=\sum_{i=1}^{K} \log \left(V_{i}\right)$, convex programming because $\left(\prod V_{i}\right)^{1 / N}$ is a convex function of $V_{i}$. \\
\hline Max-min fairness & $\begin{array}{l}\mathbb{U}(\mathbf{V})=\min _{i=1}^{K} V_{i}, \text { linear programming by introducing an auxiliary variable } u \text { and converting } \\
\text { the optimization into: } \max _{\mathbf{V}} u \text { subject to } \quad \forall_{1 \leq i \leq K}, \quad u \leq V_{i}, \quad V_{i}+\sum_{j \neq i} V_{j} \cdot \frac{I_{j, i}^{+}}{I_{i}^{m a x}} \leq M .\end{array}$ \\
\hline Auction revenue & $\begin{array}{l}\mathbb{U}(\mathbf{V})=\sum_{i=1}^{K} V_{i} B_{i}, \text { linear programming if } B_{i} \text { is a constant [3], separable programming if } \\
B_{i} \text { is a piecewise linear function of } V_{i}[1] .\end{array}$ \\
\hline
\end{tabular}

\section{Table 1: Optimus with Various Utility Functions}

When $M>>1, V_{i}$ is in general very large, and can be treated as a real number in (3). For example, when $M=1024$ [25], $V_{i}$ is usually higher than 100 , and the rounding error is less than $1 \%$. In this case, if $U(\cdot)$ is a linear or convex function of $V_{i}$, then (3) becomes a linear or convex programming problem with highly efficient solutions. Similar observation was found in [1], where finding a revenue-maximizing allocation in a $4000+$ bidder auction takes less than 90 seconds. Table 1 summarizes a set of popular utility functions and the tools used to solve them.

By solving (3), we derive the utility-maximizing $\mathbf{V}_{\text {opt }}$ subject to the new linearized interference constraints. We then apply the corresponding $\left\{\left\lfloor V_{i}\right\rfloor\right\}$ into Algorithm 1 to derive a successful allocation $\mathbb{A}$.

\subsection{Putting it All Together}

Built on the above analysis, we now describe the procedure used by Optimus to solve the original spectrum distribution problem. Specifically, Optimus consists of three steps:

Step 1) Transform the original physical interference model into a set of linear constraints among $\left\{V_{i}\right\}$, described by (2).

Step 2) Solve the utility-maximization allocation problem based on the new constraints in $\mathbf{1}$ ), derive the optimal $\mathbf{V}_{\text {opt }}$, and use Algorithm 1 to allocate spectrum bands to nodes based on $\mathbf{V}_{\text {opt }}$.

Step 3) On top of the solution from 2), apply iterative improvements based on the physical interference model for additional performance improvement. This is because the linear constraints defined by (2) are sufficient conditions for achievable volume vectors, and are thus stricter than the original constraints. The iterative improvements use the original constraint and work as follows: starting from an initial allocation derived from the previous step, iteratively allocates unused bands to nodes. In each iteration, choose a node $i$ probabilistically, and assign one more band to $i$ without making any allocated node's SINR fall below $\beta$. The probability of choosing a node $i$ is proportional to $V_{i}$. Repeat until no more bands can be allocated.

\section{PERFORMANCE BOUNDS}

In this section, we evaluate Optimus in its analytical performance bound. We focus on examining the performance bound achieved from using the linear constraints in (2) rather than the original constraints. Because the conclusion depends on the signal propagation model, we start from the most typical geometric signal propagation, and then consider arbitrary signal propagation.

Intuitively our evaluation would also depend on the utility function $U$. Using the methods of [26], we can show that the spectrum distribution problem is NPhard for typical utility function such as spectrum utilization, auction revenue, social welfare and fairness. Thus, instead of confining our findings to any specific utility function, we examine Optimus in terms of its achievable volume vector $\mathbf{V}$ compared to the optimal solution. This metric can map directly to a utility bound under a variety of utility functions, including utilization, max-min, and auction revenue.

Geometric Signal Propagation. In this case, the pathloss from node $j$ to node $i$ can be modeled as $Q_{j, i}=$ $d_{j, i}^{\alpha}$, where $d_{j, i}$ is the geographic distance from node $j$ to node $i$ 's operating area (see Definition 2, Section 2), and $\alpha \geq 2$ is the pathloss exponent. We assume the system uses linear power assignment, where the worstcase receive power in each node's area is the same: $S_{i}=$ $S$ [23]. Then when $M>>1$, Optimus performs within a constant bound $\left(\min \left\{2^{\alpha}+1,10\right\}\right)$ to the optimum:

Theorem 2. Suppose the optimal solution can achieve $\mathbf{V}$, then Optimus can achieve at least $\left\lfloor\frac{1}{\min \left\{2^{\alpha}+1,10\right\}} \mathbf{V}\right\rfloor$.

Proof. The proof uses the following lemma on the necessary condition for achievable volume vectors.

Lemma 3. Any volume vector $\mathbf{V}=\left(V_{1}, V_{2}, \cdots, V_{K}\right)$ achievable by a successful allocation, must satisfy

$$
\forall_{i}, \quad V_{i}+\sum_{j \neq i} V_{j} \cdot \frac{I_{j, i}^{+}}{I^{\text {max }}} \leq\left(\min \left\{2^{\alpha}+1,10\right\}\right) \cdot M
$$

where $I^{\max } \triangleq I_{i}^{\max }$ is uniform since $S_{i}$ is uniform.

The proof of Lemma 3 is in the Appendix.

Now it is easy to prove Theorem 2 using Lemma 3 and Theorem 1. Note that the necessary condition (4) and 
the sufficient condition (2) only differ in the coefficient of $M$. Therefore, for any allocation vector $\mathbf{V}$ achievable under $M$ bands, from Lemma 3, $\mathbf{V}$ must satisfy (4), which exactly means that $\left\lfloor\frac{1}{\min \left\{2^{\alpha}+1,10\right\}} \mathbf{V}\right\rfloor$ meets the sufficient condition (2). So Optimus can achieve $\left\lfloor\frac{1}{\min \left\{2^{\alpha}+1,10\right\}} \mathbf{V}\right\rfloor$.

From Theorem 2, we see that Optimus achieves a constant bound from the optimum achievable allocation vector. The bound is 5 for $\alpha=2,9$ for $\alpha=3$, and is no larger than 10 for any $\alpha$. Compared to existing solutions $[2,20]$, our performance bound is constant and significantly smaller.

Arbitrary Signal Propagation. With this general model, the optimization becomes much more difficult.

Theorem 3. Suppose $\mathbb{U}(\mathbf{V})=\sum_{i=1}^{K} V_{i}$, for any number of bands $M$, no polynomial time algorithm can approximate the optimal solution within a constant factor, unless $P=N P$.

Proof. Due to space limit, we only provide a sketch of the proof. It follows a standard technique that reduces the Maximum Independent Set (MIS) problem into the spectrum distribution problem. Since MIS is hard to approximate [27], so is this problem.

\section{EVALUATION}

In this section, we perform network simulations to examine the effectiveness of Optimus in the context of centralized spectrum distribution. We compare three algorithms that directly operate on the physical model.

- OPT: The optimal allocation via exhaustive search. It takes 70 hours to compute for a 40-node instance.

- Greedy: It chooses random nodes and random available bands to allocate, until no more bands can be further allocated. In general, this algorithm does not provide any performance bound. We also consider another greedy-based algorithm GAHT [2]. It uses a plane-division pattern to control the process of greedy allocation.

- Optimus: Our proposed algorithm.

The performance of all three algorithms depends heavily on the underlying RF propagation environment. Thus we consider three different environments built from propagation models and real network measurements. Table 2 summarizes the parameters used in each environment.

- GEO: It simulates the geometric propagation scenario. The pathloss from node $j$ to node $i$ can be modeled as $Q_{j, i}=d_{j, i}^{\alpha}, d_{j, i}$ is the largest geographic distance from node $j$ to node $i$ 's coverage area, and $\alpha \geq 2$ is the pathloss exponent.
For our experiments, we randomly deploy a number of nodes in a $2000 m \times 2000 m$ area. Assuming a SINR threshold $\beta=10 \mathrm{~dB}$, we adjust each node's transmission power to ensure that it has a coverage area of $50 \mathrm{~m}$ without considering interference.

- SPLAT! [28]: It is a RF simulator using sophisticated radio propagation models, incorporating both terrain and environmental factors.

For our experiments, we randomly distribute 200 nodes (APs) in a suburban area. The whole area is treated as a $200 \times 200$ grid with 40000 location points. For each point, the trace contains the corresponding GPS coordinates and the received signal strength from each AP. We treat each point as a potential user location, and associate each user with the AP from which this user receives the strongest signal. We adjust the coverage area so that each user's received signal to noise ratio is $3 \mathrm{~dB}$ above the threshold $\beta$.

- GoogleWiFi Trace [29]: This trace was collected in October 2007 [29], covering 168 APs in the Mountain View GoogleWiFi network. The trace consists of the GPS coordinates of each measured location and the set of beacons received from multiple APs at this location. Each beacon records the signal strength, noise, band ID, and AP ID. The trace does not report all the beacons transmitted by the 168 APs, because beacons received with low signals cannot be decoded correctly and thus their signal strengths are recorded as zeros.

In our experiment, we treat measured points as user locations, and use the signal strength in each beacon as the signal or interference power. We associate each user with the AP carrying the strongest signal. This produces 151 APs with at least one user associated.

The three environments exhibit very different characteristics. Table 3 summarizes the statistical distribution of $I_{j, i} / I_{i}^{\max }$, where $I_{i}^{\max }$ is the maximal interference that $\mathrm{AP} i$ and its users can sustain to guarantee $S I N R_{i} \geq \beta$. We divide the range into four cases. Given $K$ APs in total, $\left(0, \frac{1}{K-1}\right)$ is the case where accumulative interference has negligible impact since even with all other $(K-1)$ APs transmitting on the same channel, they still cannot make AP $i$ 's $S I N R_{i}$ below $\beta$. $\left[\frac{1}{K-1}, 1\right)$ is the case where accumulative interference could affect the allocation performance. From Table 3, we see that in GEO accumulative interference has significant impact. In SPLAT!, the interference power displays a higher variance, but most of them are too weak to generate impact. In GoogleWiFi, the majority of (weak) interference power are discarded due to the measurement artifact.

In SPLAT! and GoogleWiFi, most $I_{i, j}$ and $I_{j, i}$ are not equal due to irregular RF propagation. Figure 2 


\begin{tabular}{|c|c|c|c|}
\hline Parameter & GEO & SPLAT! & GoogleWiFi \\
\hline Number of bands & 100 & 200 & 100 \\
\hline Range of node $\left(d_{i, i}\right)$ & 50 meters & N/A & N/A \\
\hline Simulation area & $2000 m \times 2000 m$ & $18900 m \times 19500 m$ & $3200 m \times 4300 m$ \\
\hline Pathloss exponent $(\alpha)$ & 2,3 & 2 & N/A \\
\hline TX power $\left(P_{i}\right)$ & $5 \mathrm{dBm}$ & $23 \mathrm{dBm} / 200 \mathrm{~mW}$ & $23 \mathrm{dBm} / 200 \mathrm{~mW}$ \\
\hline Noise power $(N)$ & $-102.5 \mathrm{dBm}$ & $-98 \mathrm{dBm}$ & $-98 \mathrm{dBm}$ \\
\hline SINR threshold $(\beta)$ & $10 \mathrm{~dB}$ & $10 \mathrm{~dB}$ & $20 \mathrm{~dB}$ \\
\hline
\end{tabular}

Table 2: Network parameters used in three different propagation environments.

\begin{tabular}{|c|c|cc|cc|}
\hline & \multirow{2}{*}{ GEO } & \multicolumn{2}{|c|}{ SPLAT! } & \multicolumn{2}{c|}{ GoogleWiFi } \\
& Original & After adjustment & Original & After adjustment \\
\hline 0 & $0 \%$ & $0 \%$ & $0 \%$ & $94.59 \%$ & $92.68 \%$ \\
\hline$\left(0, \frac{1}{K-1}\right)$ & $15.04 \%$ & $71.18 \%$ & $63.69 \%$ & $0 \%$ & $0 \%$ \\
\hline$\left[\frac{1}{K-1}, 1\right)$ & $84.56 \%$ & $10.66 \%$ & $13.27 \%$ & $0.02 \%$ & $0.02 \%$ \\
\hline$[1, \infty]$ & $0.40 \%$ & $18.16 \%$ & $23.04 \%$ & $5.39 \%$ & $7.30 \%$ \\
\hline
\end{tabular}

Table 3: Distribution of $I_{j, i} / I_{i}^{\max }$ in terms of four ranges before and after replacing $I_{i, j}$ and $I_{j, i}$ with $\max \left\{I_{i, j}, I_{j, i}\right\}$. In GEO, the adjustment does not affect the interference distribution because $I_{i j}$ is symmetric. The range of $\left[\frac{1}{K-1}, 1\right)$ is where accumulative interference could impact the allocation performance, $K$ is the number of nodes.

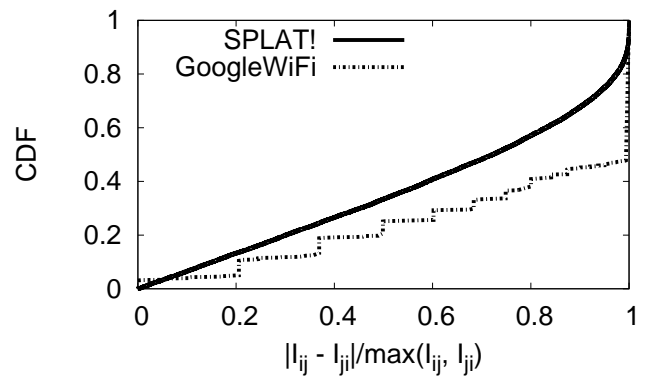

Figure 2: The level of asymmetry of $I_{i j}$ in both SPLAT! and GoolgeWiFi.

plots the CDF of the normalized difference between the two, showing that the normalized difference exceeds $70 \%$ in half the instances. Because Optimus requires $Q_{i, j}=Q_{j, i}$ which implies that $I_{i, j}=I_{j i}$ when $P_{j}=P_{i}$, we replace $I_{i, j}$ and $I_{j, i}$ with $\max \left\{I_{i, j}, I_{j, i}\right\}$ as the algorithm input. For both OPT and Greedy, we skip the adjustment. To estimate the impact of asymmetry, Table 3 also lists the interference distribution after the adjustment. Interestingly, the adjustment produces minimum impact on the interference distribution.

In the following, we present the results that compare Optimus, OPT, and Greedy. To measure the allocation performance, we set the optimization objective as the spectrum utilization, $U=\sum_{i=1}^{K} V_{i}$, and evaluate the solutions by the utility (spectrum utilization). In the following, we refer to $U_{x}$ as the spectrum utilization generated by the scheme $x$.

\subsection{Optimus vs. OPT}

To examine Optimus's efficiency, we first compare it to OPT. Since OPT requires exponential complexity, we use small-scale networks with 40 nodes and 100 randomly generated topologies. We plot the performance gap to OPT, defined by:

$$
\sigma=\mathbb{U}_{\text {Optimus }} / \mathbb{U}_{O P T},
$$

where $\mathbb{U}_{O P T}$ represents the utilization of OPT with the original $I_{i, j}$ value as input. To further explore the impact of the asymmetry of $I_{i, j}$ in both SPLAT! and GoolgeWiFi, we also evaluate $\sigma_{S y m}$, the gap to OPT by replacing both $I_{i, j}$ and $I_{j, i}$ with $\max \left(I_{i, j}, I_{j, i}\right)$.

GEO. Figure 3 (a) shows the CDFs for $\alpha=2$ and $\alpha=3$ across 100 topologies. We see that Optimus consistently achieves more than $90 \%$ of the utilization compared to OPT. This is because Optimus applies the two design principles in Sec. 3, avoiding most performance traps. This result demonstrates the efficiency of Optimus's globalized optimization and its performance guarantee.

Another key observation comes from comparing the results of $\alpha=2$ and $\alpha=3$. The performance gap of Optimus is higher when $\alpha=3$. This is because Optimus becomes more conservative as $\alpha$ increases. A larger $\alpha$ means that the interference at each node will more likely be dominated by the strongest interferer and become localized. Thus by weighting $I_{j, i}$ by $V_{j}$ (from (2)), Optimus intends to overestimate the effect of accumulative interference, leading to conservative allocations.

SPLAT! and GoogleWiFi. From the results in Figure 3 (b) and (c), we arrive at two key observations. First, in average Optimus achieves $80 \%-85 \%$ utilization of OPT, demonstrating its efficiency under irreg- 


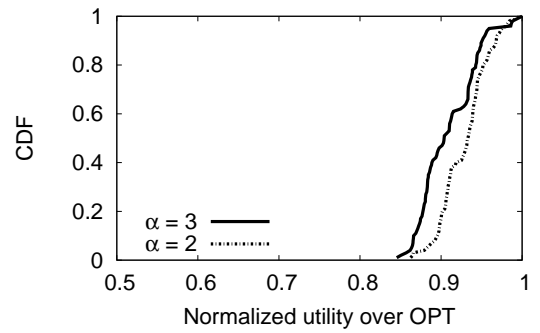

(a) Geometric $\alpha=2,3$

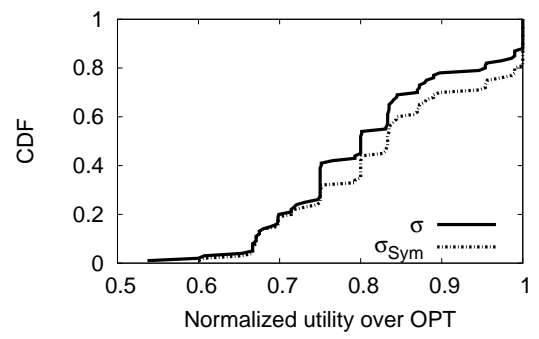

(b) SPLAT!

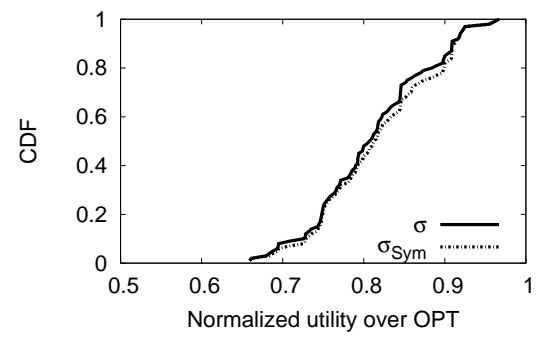

(c) GoogleWiFi

Figure 3: Comparing Optimus to OPT in small-scale networks. $x$-axis denotes $\sigma=\mathbb{U}_{O p t i m u s} / \mathbb{U}_{O P T}$, the gap to OPT with original $I_{i, j}$, or $\sigma_{S y m}=\mathbb{U}_{O p t i m u s} / \mathbb{U}_{O P T_{S y m}}$, the gap to OPT with symmetric $I_{i, j}$.

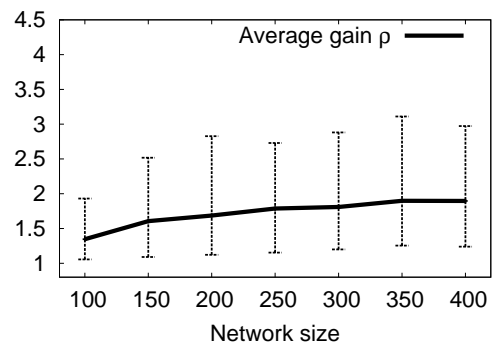

(a) Geometric $\alpha=3$

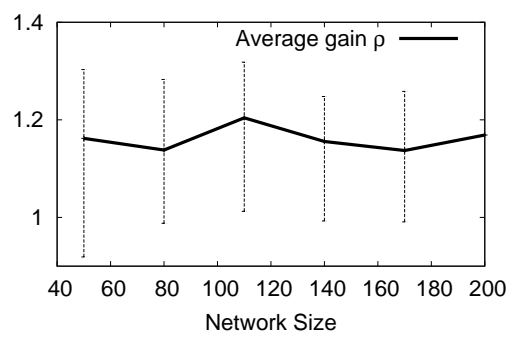

(b) SPLAT!

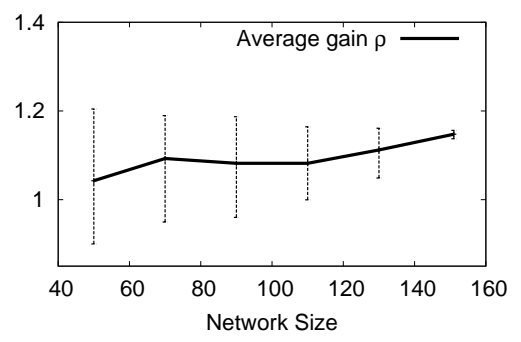

(c) GoogleWiFi

Figure 4: Comparing Optimus and Greedy in large scale networks. $y$-axis denotes $\rho$, the gain of Optimus over Greedy and $90 \%$ confidence interval.

ular propagation conditions. Second, the performance gap varies in a larger range than that in GEO. This is mainly because the propagation conditions in these two cases are close to GEO with larger $\alpha$, where the interference is either very strong or very weak (Table (3)). In these cases, Optimus's interference constraint transformation becomes too conservative.

One might also suspect that the increased performance gap is also triggered by the asymmetry of $I_{i, j}$. To examine this effect indirectly, we examine the gap between Optimus and $\mathrm{OPT}_{\text {sym }}$ where for both algorithms the $I_{i j}$ is symmetric. Interestingly, the performance gap remains similar to that to OPT, showing that the asymmetry does not have a large impact. This can be explained by the interference distribution in Table (3) where for both SPLAT! and GoogleWiFi, converting $I_{i j}$ and $I_{j i}$ does not produce significant change to the interference distribution.

\subsection{Optimus vs. Greedy}

To understand Optimus's advantage over existing greedy solutions, we use following performance metric:

$$
\rho=\mathbb{U}_{\text {Optimus }} / \mathbb{U}_{\text {Greedy }} .
$$

GEO. We start by considering a network of 100 nodes. Table 4 lists the distribution of $\rho$ over 100 random topologies, where Optimus achieves an average gain of 1.5 . In $17 \%$ of the cases, the gain exceeds 2 .

\begin{tabular}{|c|c|c|c|c|}
\hline$\rho$ & {$[1,1.2)$} & {$[1.2,1.5)$} & {$[1.5,2)$} & $>2$ \\
\hline Occurance & $11 \%$ & $38 \%$ & $34 \%$ & $17 \%$ \\
\hline
\end{tabular}

Table 4: The distribution of $\rho$ over 100 random topologies.

In Figure 4 (a) we plot the gain of Optimus to Greedy as a function of the network size. We make three observations. First, Optimus consistently outperforms Greedy with an average gain of $1.5-2$. The gain increases to a factor of 3 for $5 \%$ of the cases. Second, the gain increases slightly with the network size, from 1.5 for 100 nodes to $2+$ for 400 nodes. This is because as the network size increases, the impact of accumulative interference elevates, and a "bad" greedy decision is more likely to prevent efficient allocation. In contrast, Optimus is not affected by the increased level of interference because it explicitly considers the accumulative interference across the entire network.

SPLAT! and GoolgeWiFi. Given the original network deployments in these two cases, we keep the network density the same, and generate networks with various sizes by sampling different areas. For each network size, the result is averaged over 50 sampled areas.

Figures 4(b)-(c) plot the gain $\rho$ in its average value and the $90 \%$ confidence interval. We obtain two key observations. First, the average gain in both cases is 


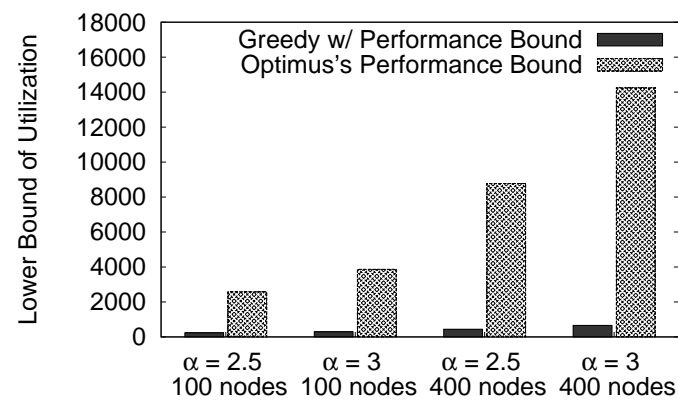

Figure 5: Optimus outperforms the greedy algorithm [2] in terms of performance lower bound. Both algorithms provide provable approximation bounds without applying additional improvements.

smaller compared to that in GEO. This is because both SPLAT! and GoogleWiFi display almost binary interference conditions. The interference power is either very strong or very weak (see Table 3 ). In this case, Greedy performs well because in each allocation step, it can estimate the impact of the interference to future step. However, Optimus still achieves 10\%-20\% gain over Greedy. Second, the gain in GoolgeWiFi is slightly smaller compared to that in SPLAT!. This is due to the measurement artifact in GoogleWiFi trace, where most of the weak $I_{i, j}$ were treated as zeros (Table 3). This further diminishes the impact of accumulative interference and thus the gain of Optimus.

\subsection{Optimus vs. Greedy with Performance Bound}

To evaluate the effectiveness of Optimus's performance bound, we compare Optimus to GAHT [2], a greedy solution that provides performance guarantee but does not perform additional iterative improvements to saturate the allocation. For a fair comparison, we use Optimus-Lite, i.e. Optimus without the iterative improvement, but achieves the same analytical performance bound. GAHT achieves a performance bound as a function of the pathloss exponent $\alpha$ and the SINR threshold $\beta$. The bound is larger than 80 under our default settings with $\alpha=2.5$. We note that GAHT cannot be applied to $\alpha=2$. In contrast, Optimus achieves a bound of 6.7 under the same settings (see Theorem 2). To further understand the performance bound, we compare the actual spectrum utilization of Optimus-Lite and GAHT under GEO model in Figure 5. We observe that Optimus-Lite has an average gain factor of 11 under both $\alpha=2.5$ and 3.5.

\section{RELATED WORK}

We classify the most relevant literatures on the physical model into the following three categories.

First, recent works on link scheduling have considered the physical interference models, and have developed efficient algorithms that achieve provable bounds from the optimum $[20,21]$. Some also jointly considered time scheduling with power control and achieved approximation bounds $[10,23]$. As discussed earlier, the problem considered in this paper differs significantly from the link scheduling problem because $V_{i}$ is unknown prior to the optimization. Consequently, we adopt a very different approach (constraint transformation) to solve the new problem. A recent work [11] also used linearization to solve the problem of joint scheduling and routing for capacity-maximization. Our work applies this general approach but focuses on deriving a linearization suited for spectrum distribution. Our solution also achieves constant bounds that remain small for different $\alpha$ and network sizes. On the other hand, we note that despite the key differences, these two problems share similar hardness results: both can be approximated within a constant factor under geometric signal propagation but become inapproximable under arbitrary signal propagation [21].

Second, another research problem using physical interference models is the resource allocation in cellular networks. As mentioned before, most solutions in this category apply linear/convex programming based on a simplified physical interference model such as using a regulated topology $[12,13]$, or only considering a single dominant interferer for each node [19], or applying a graph interference model [16]. Several works have also jointly considered channel allocation with multi-carrier diversity, rate selection and power control. Due to its complexity, most solutions focus on heuristic-based algorithms (e.g. greedy) without considering approximation guarantees $[14,15,17]$. Different from these cellular network solutions, the main contribution of Optimus is to discover a linear transformation of the physical interference model under unregulated topology, and to analytically show that it guarantees an achievable allocation and provides a constant approximation ratio.

Third, a recent work on spectrum distribution [2] proposes two efficient greedy allocation algorithms. Assuming geometric signal propagation and uniform transmit power, these algorithms achieve a provable approximation ratio. But the ratio becomes unbounded when the pathloss exponent $\alpha$ approaches 2. While our problem formulation is inspired by this work, Optimus applies a different mechanism (i.e. global planning) and achieves a constant $(\leq 10)$ approximation ratio. In Section 6 we also verify the gain of Optimus using network simulations. Finally, a prior work [8] proposed an indirect solution to build a conflict graph from the physical interference model, and then apply a graph-based allocation algorithm. This work, on the other hand, shows that the graph optimization is a fundamentally hard problem. In this paper, we propose an alterative solution that directly operates over the physical interference model. 


\section{CONCLUSION AND FUTURE WORK}

In this paper we consider the problem of spectrum distribution using physical interference models. We propose a new line of algorithms using interference transformation that perform within a constant bound from the optimum and support a wide-range of optimization goals. Experiments show that in average our solution outperforms existing solutions by $20-50 \%$ in utilization, and is within $20 \%$ gap from the optimal solution.

In its current design, Optimus operates on instantaneous SINR measurements or assumes that SINR measurements are static over time. In practice the signal propagation experiences shadowing and fading, so SINRs are time-varying. Therefore, how to measure the signal and interference levels reliably and how to compensate for their time dynamics are both interesting open research problems.

In Optimus we use a threshold model to map from SINR to the channel quality, in accordance with the widely-accepted formalization of the physical model [22]. This assumption also makes the optimization problem tractable. We notice that a recent work [30] in link scheduling has used a more realistic model, namely the graded SINR model, and designed efficient scheduling algorithms with constant approximation bounds. Inspired by this effort, it is an interesting future work to extend Optimus under the graded SINR model, possibly by finding a constraint transformation suited for this model.

\section{Acknowledgement}

This research was supported by the National Science Foundation under grants CNS-0915699, CNS-0832090 and CNS-0721961, and by a research gift from Samsung.

\section{REFERENCES}

[1] S. Gandhi, C. Buragohain, L. Cao, H. Zheng, and S. Suri, "A general framework for wireless spectrum auctions," in Proc. of IEEE DySPAN, 2007.

[2] A. Subramanian, M. Al-ayyoub, H. Gupta, S. Das, and M. Buddhikot, "Near-optimal dynamic spectrum allocation in cellular networks," in Proc. of IEEE DySPAN, 2008.

[3] X. Zhou, S. Gandhi, S. Suri, and H. Zheng, "eBay in the sky: strategy-proof wireless spectrum auctions," in Proc. of MobiCom, 2008.

[4] X. Zhou and H. Zheng, "Trust: A general framework for truthful double spectrum auctions," in Proc. of INFOCOM, 2009.

[5] E. Rozner, Y. Mehta, A. Akella, and L. Qiu, "Traffic-aware channel assignment in enterprie wireless networks," in Proc. of ICNP, 2007.

[6] T. Moscibroda, R. Chandra, Y. Wu, S. Sengupta, P. Bahl, and Y. Yuan, "Load-aware spectrum distribution in wireless LANs," in Proc. of ICNP, 2008.

[7] L. Cao and H. Zheng, "SPARTA: Stable and efficient spectrum access in next generation dynamic spectrum networks," in Proc. of INFOCOM, 2008.

[8] L. Yang, L. Cao, and H. Zheng, "Physical interference driven dynamic spectrum management," in Proc. of IEEE DySPAN, 2008.

[9] J. Gronkvist and A. Hansson, "Comparison between graph-based and interference-based STDMA scheduling," in Proc. of MobiHoc, 2001.

[10] T. Moscibroda, R. Wattenhofer, and Y. Weber, "Protocol Design Beyond Graph-Based Models," in Proc. of HotNets, 2006.

[11] D. Chafekar, V. S. A. Kumar, M. V. Marathe, S. Parthasarathy, and A. Srinivasan, "Approximation algorithms for computing capacity of wireless networks with SINR constraints." in Proc. of INFOCOM, 2008.

[12] H. Kim, Y. Han, and J. Koo, "Optimal subchannel allocation scheme in multicell OFDMA systems," in Proc. of VTC, 2004.

[13] W. C. Lee, "Spectrum efficiency in cellular," IEEE Trans. Vehicular Technology, vol. 38, no. 2, pp. 69-75, 1989.

[14] I. Koutsopoulos and L. Tassiulas, "Channel state-adaptive techniques for throughput enhancement in wireless broadband networks," in Proc. of INFOCOM, 2001.

[15] Z. Han, F. R. Farrokhi, Z. Ji, and K. J. R. Liu, "Capacity optimization using subspace method over multicell OFDMA networks," in Proc. of WCNC, 2004.

[16] M. Hellebrandt, F. Lambrecht, R. Mathar, T. Niessen, and R. Sta, "Frequency allocation and linear programming," in Proc. of VTC, 1999.

[17] A. Abrardo, A. Alessio, P. Detti, and M. Moretti, "Radio resource allocation problems for OFDMA cellular systems." Computers $\& 3$ Operations Research, vol. 36, no. 5, pp. 1572-1581, 2009.

[18] K. Jain, J. Padhye, V. Padmanabhan, and L. Qiu, "Impact of interference on multi-hop wireless network performance," in Proc. of MobiCom, 2003.

[19] G. Li and H. Liu, "Downlink radio resource allocation for multi-cell OFDMA system," IEEE Trans. Wireless Commun., vol. 5, no. 12, pp. 3451-3459, 2006.

[20] G. Brar, D. M. Blough, and P. Santi, "Computationally efficient scheduling with the physical interference model for throughput improvement in wireless mesh networks," in Proc. of MobiCom, 2006.

[21] O. Goussevskaia, R. Wattenhofer, M. M. Halldorsson, and E. Welzl, "Capacity of arbitrary wireless networks," in Proc. of INFOCOM, 2009. 
[22] P. Gupta and P. R. Kumar, "The capacity of wireless networks," IEEE Transactions on Information Theory, vol. 46, no. 2, pp. 388-404, 2000 .

[23] T. Moscibroda, Y. A. Oswald, and R. Wattenhofer, "How Optimal are Wireless Scheduling Protocols?" in Proc. of INFOCOM, 2007.

[24] C. Peng, H. Zheng, and B. Y. Zhao, "Utilization and fairness in spectrum assignemnt for opportunistic spectrum access," Mobile Networks and Applications (MONET), vol. 11, pp. 555-576, May 2006.

[25] R. Rajbanshi, A. M. Wyglinski, and G. J. Minden, "An efficient implementation of NC-OFDM transceivers for cognitive radios," in Crown Com, 2006.

[26] O. Goussevskaia, Y. A. Oswald, and R. Wattenhofer, "Complexity in geometric SINR," in Proc. of MobiHoc, 2007.

[27] J. Hastad, "Clique is hard to approximate within $n^{1-\epsilon}, "$ in Proc. of FOCS, 1996.

[28] SPLAT!, "A terrestrial rf path analysis application for linux/unix," http://www.qsl.net/kd2bd/splat.html.

[29] J. Robinson, R. Swaminathan, and E. Knightly, "Assessment of urban-scale wireless networks with a small number of measurements," in Proc. of MobiCom, September 2008.

[30] P. Santi, R. Maheshwari, G. Resta, S. Das, and D. M. Blough, "Wireless link scheduling under a graded SINR interference model," in FOWANC, 2009.

\section{APPENDIX}

\section{Proof of Lemma 1}

For every allocation $\mathbb{A}$, define a metric $F(\mathbb{A})$ (Recall that $Q_{i, j}=Q_{j, i}$ is the pathloss between $i$ and $j$ ):

$$
F(\mathbb{A})=\sum_{m=1}^{M} \sum_{1 \leq i<j \leq N} a_{m, i} \cdot a_{m, j} \cdot \frac{P_{i} \cdot P_{j}}{Q_{i, j}} .
$$

When node $i$ adjusts from a band $m$ to a band $m^{\prime}$, changing $\mathbb{A}$ to $\mathbb{A}^{\prime}$ only if $\omega_{m^{\prime}, i}<\omega_{m, i}$. That is,

$$
\sum_{j \neq i} a_{m^{\prime}, j} \cdot I_{j, i}<\sum_{j \neq i} a_{m, j} \cdot I_{j, i} .
$$

Multiply both sides by $P_{i}$, and recall $I_{j, i}=P_{j} / Q_{i, j}$,

$$
\sum_{j \neq i} a_{m^{\prime}, j} \frac{P_{i} \cdot P_{j}}{Q_{i, j}}<\sum_{j \neq i} a_{m, j} \frac{P_{i} \cdot P_{j}}{Q_{i, j}} .
$$

Because $\mathbb{A}^{\prime}$ differs from $\mathbb{A}$ only by adding $m^{\prime}$ to $i$ and removing $m$ from $i$, we have

$$
F(\mathbb{A})-F\left(\mathbb{A}^{\prime}\right)=\sum_{j \neq i} a_{m, j} \frac{P_{i} \cdot P_{j}}{Q_{i, j}}-\sum_{j \neq i} a_{m^{\prime}, j} \frac{P_{i} \cdot P_{j}}{Q_{i, j}}>0
$$

This shows that $F(\mathbb{A})$ decreases strictly after an adjustment. Because the number of distinct allocations is finite, the proposed adjustments will converge.

\section{Proof of Lemma 2}

For each $\left\{V_{i}\right\}_{1 \leq i \leq K}$ satisfying (2), let $\mathbb{A}$ represent the spectrum allocation when Algorithm 1 converges. We need to show the allocation is successful, i.e., $\forall_{m, i}$,

$$
\text { If } a_{m, i}=1 \text {, then } S I N R_{m, i} \triangleq \frac{S_{i}}{\sum_{j \neq i} a_{m, j} \cdot I_{j, i}+N} \geq \beta \text {. }
$$

Let $\omega_{m, i}=\sum_{j \neq i} a_{m, j} \cdot I_{j, i}$ represent the aggregate interference at node $i$ on band $m$, and recall the definition of $I_{i}^{\max }$ in (2). It is easy to see that $S I N R_{m, i} \geq \beta$ is equivalent to $\omega_{m, i} \leq I_{i}^{\max }$. So we are left to prove:

$$
\text { If } a_{m, i}=1 \text {, then } \omega_{m, i} \leq I_{i}^{\max } \text {. }
$$

Let us call a band $m$ blocked for node $i$ if $\omega_{m, i}>I_{i}^{\max }$. To show that $\mathbb{A}$ satisfies (8), we only need to show:

Claim 4. For any node $i$, there are at least $V_{i}$ bands that are not blocked.

Claim 4 is suffice to imply (8) since Algorithm 1 implies that a node always prefers unblocked bands to blocked ones. With at least $V_{i}$ unblocked bands, $i$ obtains enough unblocked bands when Algorithm 1 converges. In the rest of the proof we prove Claim 4 . We bound the maximum number of bands that could be blocked by interference.

We divide the blocked band set $\Phi$ into $\Phi_{1}$ and $\Phi_{2}$.

$$
\begin{aligned}
\Phi= & \left\{m \mid \omega_{m, i}>I_{i}^{\max }\right\} \\
= & \left\{m \mid \exists_{j}, a_{m, j}=1 \wedge I_{j, i}>I_{i}^{\max }\right\} \cup \\
& \left\{m \mid\left(\forall_{j}, a_{m, j}=0 \vee I_{j, i} \leq I_{i}^{\max }\right) \wedge\left(\omega_{m, i}>I_{i}^{\max }\right)\right\} \\
\triangleq & \Phi_{1} \cup \Phi_{2}
\end{aligned}
$$

Intuitively, $\Phi_{1}$ contains the bands blocked by a "heavy" interferer while $\Phi_{2}$ includes the bands blocked by accumulative interference from "light" interferers.

We now show that the sizes of $\Phi_{1}$ and $\Phi_{2}$ are bounded. First, it is obvious that $\left|\Phi_{1}\right| \leq \sum_{j \neq i, I_{j, i}>I_{i}^{\max }} V_{j}$. Next, to bound $\left|\Phi_{2}\right|$, we have:

$$
\begin{aligned}
& \sum_{m \in \Phi_{2}} \omega_{m, i}=\sum_{m \in \Phi_{2}} \sum_{j \neq i} a_{m, j} I_{j, i}=\sum_{j \neq i} \sum_{m \in \Phi_{2}} a_{m, j} I_{j, i} \\
& =\sum_{j \neq i, I_{j, i} \leq I_{i}^{\max }} I_{j, i} \sum_{m \in \Phi_{2}} a_{m, j} \leq \sum_{j \neq i, I_{j, i} \leq I_{i}^{\max }} I_{j, i} V_{j} .
\end{aligned}
$$

From the above equation and the fact that any band $m \in \Phi_{2}$ must satisfy $\omega_{m, i}>I_{i}^{\max }$, the number of bands in $\Phi_{2}$ is bounded: $\left|\Phi_{2}\right| \leq \frac{1}{I_{i}^{\text {max }}} \sum_{j \neq i, I_{j, i} \leq I_{i}^{\max }} I_{j, i} V_{j}$. Thus, the number of blocked bands is at most

$$
\begin{array}{r}
|\Phi|=\left|\Phi_{1}\right|+\left|\Phi_{2}\right| \leq \sum_{j \neq i, I_{j, i}>I_{i}^{\text {max }}} V_{j}+\sum_{j \neq i, I_{j, i} \leq I_{i}^{\max }} \frac{I_{j, i}}{I_{i}^{\text {max }}} \cdot V_{j} \\
=\sum_{j \neq i} \cdot \frac{I_{j, i}^{+}}{I_{i}^{\text {max }}} \cdot V_{j}(\text { by }(2)) \leq M-V_{i} \text { (by (2)). }
\end{array}
$$


This shows that there are at least $V_{i}$ unblocked bands, which implies Claim 4 and completes our proof.

\section{Proof of Lemma 3}

Suppose the volume vector $\mathbf{V}=\left(V_{1}, V_{2}, \cdots, V_{K}\right)$ is achieved by a successful allocation $\mathbb{A}$. We prove that (4) is true. Define $\omega_{m, i}^{+} \triangleq \sum_{j \neq i} a_{m, j} \cdot I_{j, i}^{+}$. We have

$$
\begin{aligned}
& \sum_{j \neq i} V_{j} \cdot I_{j, i}^{+}=\sum_{j \neq i} \sum_{m=1}^{M} a_{m, j} \cdot I_{j, i}^{+} \\
= & \sum_{m=1}^{M} \sum_{j \neq i} a_{m, j} \cdot I_{j, i}^{+}=\sum_{m=1}^{M} \omega_{m, i}^{+}=\sum_{a_{m, i}=1} \omega_{m, i}^{+}+\sum_{a_{m, i}=0} \omega_{m, i}^{+} \\
\leq & V_{i} \cdot I^{\max }+\left(M-V_{i}\right) \cdot\left(\min \left\{2^{\alpha}+1,10\right\}\right) I^{\max } \\
\leq & \left(\min \left\{2^{\alpha}+1,10\right\}\right) M \cdot I^{\max }-V_{i} \cdot I^{\max } .
\end{aligned}
$$

The first inequality in the above follows from two facts. First, by the following Lemma $4, \omega_{m, i}^{+} \leq I^{\max } \cdot \min \left\{2^{\alpha}+\right.$ $1,10\}$. Next, it is clear that $\omega_{m, i}^{+} \leq I^{\max }$ if $a_{m, i}=1$ since the allocation $\mathbb{A}$ is successful. With the following proof of Lemma 4, we conclude the proof of Lemma 3.

Lemma 4. Under geometric radio propagation, for any successful allocation $\mathbb{A}$, any band $m$ and node $i$,

$$
\omega_{m, i}^{+} \triangleq \sum_{j \neq i} a_{m, j} \cdot I_{j, i}^{+} \leq I^{\max } \cdot \min \left\{2^{\alpha}+1,10\right\} .
$$

Proof. We prove (9) in two steps.

Step 1: First, we prove $\omega_{m, i}^{+} \leq\left(2^{\alpha}+1\right) I^{\max }$. Consider the allocation on band $m$. If $i$ is allocated $\left(a_{m, i}=\right.$ 1 ), we have $\omega_{m, i}^{+} \leq I^{\max }$ since $\mathbb{A}$ is successful. Now suppose $i$ is not allocated $\left(a_{m, i}=0\right)$. Let $k$ be the allocated node closest to $i$ (Fig. 6). Since $\mathbb{A}$ is successful, $\omega_{m, k}^{+} \leq I^{\max }$, i.e.

$$
\sum_{j \neq k} a_{m, j} \cdot I_{j, k}^{+} \leq I^{\max } .
$$

We claim that for any allocated node $j \neq i, k, I_{j, i}^{+} \leq$ $2^{\alpha} \cdot I_{j, k}^{+}$. To show this, note that node $j$ must be outside the circle in Fig. 6 since $k$ is the closest allocated node to $i$. By geometry, any node $j$ outside the circle must have $d_{j, i} \geq d_{j, k} / 2$ (the equality is reached when $k$ is at position $X)$. Thus $I_{j, i} \leq 2^{\alpha} I_{j, k}$, which implies $I_{j, i}^{+} \leq$ $2^{\alpha} I_{j, k}^{+}$. Combining this with (10),

$$
\begin{aligned}
\omega_{m, i}^{+} & =\sum_{j \neq i} a_{m, j} \cdot I_{j, i}^{+}=I_{k, i}^{+}+\sum_{j \neq i, k} a_{m, j} \cdot I_{j, i}^{+} \\
& \leq I^{\max }+\sum_{j \neq i, k} a_{m, j} \cdot 2^{\alpha} I_{j, k}^{+} \\
& \leq I^{\max }+2^{\alpha} \cdot \sum_{j \neq k} a_{m, j} I_{j, k}^{+}\left(\text {notice } a_{m, i}=0\right) \\
& \leq\left(2^{\alpha}+1\right) I^{\max } \cdot(\text { by }(10))
\end{aligned}
$$

Step 2: Next, we prove $\omega_{m, i}^{+} \leq 10 I^{\max }$. Similar to Step 1 , if $i$ is allocated $\left(a_{m, i}=1\right)$, the claim is straightforward. Now suppose $i$ is not allocated $\left(a_{m, i}=0\right)$.
Let node $k$ be the allocated node closest to $i$. We divide the whole plane into 5 fan-like areas as shown in Fig. 6 , where $\angle 1=120^{\circ}$ with node $k$ in its middle, and $\angle 2=\angle 3=\angle 4=\angle 5=60^{\circ}$. Note that a similar planedivision pattern is also used in [21] to bound the number of nodes with certain properties. Instead, we use it to bound the accumulative interference for a node.

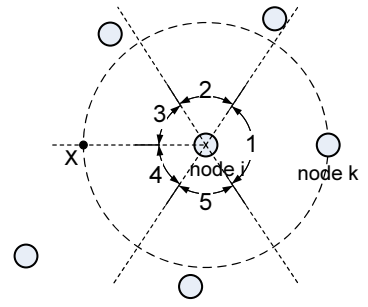

Figure 6: Analyzing the upper bound of $\omega_{m, i}^{+}$ using a similar technique of [21].

For each area, we find the allocated node closest to $i$ (Fig. 6). An important property of the selected nodes is that in each area, any allocated node will generate more interference to the selected node than to $i$. Define set $\Psi$ to be the collection of these nodes. Clearly $|\Psi| \leq 5$. Let $\mathcal{T}=\left\{j \mid j \notin \Psi \wedge a_{m, j}=1\right\}$ be the set of allocated nodes not in $\Psi$. By geometry, any node in $\mathcal{T}$ generates more interference to some node in $\Psi$ than to $i$. Formally, $\forall_{j} \in \mathcal{T}, \quad \exists_{q} \in \Psi$ s.t. $I_{j, q} \geq I_{j, i}$, which also implies $I_{j, q}^{+} \geq I_{j, i}^{+}$from its definition. Thus we have

$$
\sum_{j \in \mathcal{T}} I_{j, i}^{+} \leq \sum_{q \in \Psi} \sum_{j \in \mathcal{T}} I_{j, q}^{+} \leq \sum_{q \in \Psi} \omega_{m, q}^{+} \leq 5 I^{\max } .
$$

The last inequality holds from $\omega_{m, q}^{+} \leq I^{\max }$ and $|\Psi| \leq$ 5. With this we reach the conclusion in Step 2: $\omega_{m, i}^{+}=$ $\sum_{j \in \Psi} I_{j, i}^{+}+\sum_{j \in \mathcal{T}} I_{j, i}^{+} \leq 5 I^{\max }+5 I^{\max }=10 I^{\max }$. 